\title{
Chemosensitivity of lung cancer: Differences between the primary lesion and lymph node metastasis
}

\author{
YOSHIMASA MANIWA $^{1}$, MASAHIRO YOSHIMURA ${ }^{1,2}$, SHOTARO HASHIMOTO ${ }^{1}$, \\ MASAHIKO TAKATA ${ }^{1}$ and WATARU NISHIO ${ }^{1}$ \\ ${ }^{1}$ Division of Thoracic Surgery, Kobe University Graduate School of Medicine, Kobe; \\ ${ }^{2}$ Department of Respiratory Surgery, Hyogo Cancer Center, Hyogo, Japan
}

Received September 30, 2009; Accepted December 23, 2009

DOI: 10.3892/ol_00000061

\begin{abstract}
In this study, chemosensitivity tests were performed on both primary lesions (PLs) and lymph node metastases (LMs) from surgically resected non-small cell lung cancer (NSCLC). Differences between the results obtained were evaluated. Operative specimens were obtained from 13 patients with NSCLC [6 with squamous cell carcinoma (SQ) and 7 with adenocarcinoma (AD)] whose lymph nodes were confirmed to be positive for metastasis. Both the PL and LM from the same patient were examined immediately after resection. The collagen gel droplet-embedded culture drug sensitivity test (CD-DST) was used as the chemosensitivity test against six anticancer drugs [5-fluorouracil (5-FU), cisplatin, gemcitabine, docetaxel, vinorelbine and SN-38 (an active metabolite of irinotecan)]. When the growth rate, determined by the $\mathrm{T} / \mathrm{C}$ ratio $(\mathrm{T}$, signal for viable cells in the treated group and $\mathrm{C}$, signal in the control) was less than $50 \%$, the tumor cells were considered to be sensitive to the drug. Only in 4 cases (2 SQ and 2 AD) was the chemosensitivity of the primary lesion identical to that of LM. In the SQ cases, chemosensitivity of the primary lesions to 5-FU tended to be consistent with that of LMs. In contrast, the primary lesions in 4 of the $7 \mathrm{AD}$ cases were negative for chemosensitivity to 5-FU; however, LMs were sensitive. In many cases, the chemosensitivity of the PLs to each anticancer drug differed from that of the LMs. In conclusion, both primary and metastatic tumors should be examined to ensure maximum clinical efficacy of in vitro drug-sensitivity testing for adjuvant chemotherapy after complete resection of $\mathrm{n} 1$ and n2 NSCLC.
\end{abstract}

\section{Introduction}

The effectiveness of chemotherapy directly affects the prognosis of advanced lung cancer. Adjuvant chemotherapy is

Correspondence to: Dr Yoshimasa Maniwa, Division of Thoracic Surgery, Kobe University Graduate School of Medicine, Kobe, Japan E-mail: maniwa@med.kobe-u.ac.jp

Key words: lung cancer, drug-sensitivity test, lymph node metastasis, adjuvant chemotherapy expected to contribute to the improvement of the prognosis of patients who undergo curative surgical treatments. The clinical outcome of chemotherapy against non-small cell lung cancer (NSCLC) has improved since new drugs have been developed and their combination therapies have been established. However, these drugs are not always equally efficacious in all NSCLC patients. A drug that has curative effects on some patients has sometimes been observed to cause only side effects in other patients. These findings suggest the need for the appropriate selection of drugs separately for each individual patient. Certain in vitro chemosensitivity tests have been established for the identification of an effective anticancer drug for individual cases (1-3), and the efficacy of these assays has been described in many clinical reports on lung cancer (4-9) and other malignancies (10-15).

Anticancer drugs are expected to be effective not only against primary but also against metastatic lesions. Specifically, adjuvant chemotherapy, after complete resection of lung cancer, targets putative dormant metastasis. However, in certain clinical cases the chemosensitivity of metastatic lesions differs from that of primary lesions. This appears to be normal since the pathological features of the primary lesion and lymph node metastasis (LM) of lung cancer are frequently different from each other. In such cases, chemosensitivity testing for the primary lesion may be useless in controlling dormant metastatic lesions.

We performed chemosensitivity tests for both primary lesions and LMs obtained during the surgery of NSCLC patients and compared them in order to evaluate the test for surgically resected metastatic lesions.

\section{Materials and methods}

Patients and samples. Operative specimens were obtained from 13 patients with NSCLC [6 with squamous cell carcinoma (SQ) and 7 with adenocarcinoma (AD)] whose lymph nodes were confirmed to be positive for metastasis by frozen section. All of the patients consented to participate in this study.

A block of cancer tissue, 5-10 $\mathrm{mm}$ in diameter, was obtained with aseptic manipulation immediately following resection, and stored at $4^{\circ} \mathrm{C}$ (primary lesion; PL). A lymph node obtained from the same patient was swollen and metastasis was suspected. The lymph node was divided into 
Table I. The exposure condition of anticancer drugs.

\begin{tabular}{lcc}
\hline Drugs & Concentration $(\mu \mathrm{g} / \mathrm{ml})$ & Exposure time \\
\hline 5 -FU & 1.00 & 7 days \\
CDDP & 0.20 & $24 \mathrm{~h}$ \\
GEM & 0.03 & $24 \mathrm{~h}$ \\
TXT & 0.10 & $24 \mathrm{~h}$ \\
VNR & 0.05 & $24 \mathrm{~h}$ \\
SN38 & 0.10 & $24 \mathrm{~h}$ \\
\hline
\end{tabular}

5-FU, 5-fluorouracil; CDDP, cisplatin; GEM, gemcitabine; TXT, docetaxel; VNR, vinorelbine; SN38, active metabolite of irinotecan (CPT-11).

three sections; one was stored in the frozen section and was confirmed to be cancerous, and the two remaining sections, which were $>0.5 \mathrm{~mm}^{3}$ in size, were stored at $4^{\circ} \mathrm{C}(\mathrm{LM})$.

In vitro chemosensitivity test. We initiated the collagen gel droplet-embedded culture drug sensitivity test (CD-DST) within $36 \mathrm{~h}$ after resection. This is one of the growth assays described by Kobayashi et al (3) as a chemosensitivity test. Each sample was minced finely using a scalpel or razor blade and digested in a cell dispersion enzyme solution (EZ; Nitta Gelatin Inc., Osaka, Japan) for $2 \mathrm{~h}$. The dispersed cancer cells were treated with ethylene glycol tetra-acetic acid (EGTA)trypsin and filtered through a $200-\mu \mathrm{m}$ nylon mesh. The cells were then incubated in a collagen gel-coated flask (CG-flask; Nitta Gelatin Inc.) containing preculture medium (PCM-1; Nitta Gelatin Inc.) with $10 \%$ fetal bovine serum (FBS) at $37^{\circ} \mathrm{C}$ in $5 \% \mathrm{CO}_{2}$ overnight.

Only the viable cancer cells that adhered to the collagen gel were collected, treated again with EGTA-trypsin and filtered through a $125-\mu \mathrm{m}$ nylon mesh.

Type I collagen (Cell Matrix Type CD; Nitta Gelatin Inc.), 10X F-12 medium and reconstruction buffer were added to ice water at a ratio of 8:1:1. The prepared cancer cell suspension was added to the mixed collagen solution with a final density of $1 \times 10^{5}$ cells $/ \mathrm{ml}$. Three drops of the collagen-cell mixture (30 $\mu \mathrm{l} / \mathrm{drop})$ were placed in each well of 6 -well plates and in a $35-\mathrm{mm}$ dish and were left to set at $37^{\circ} \mathrm{C}$ in a $\mathrm{CO}_{2}$ incubator. The final concentration was $3 \times 10^{3}$ cells/droplet. One hour later, $3 \mathrm{ml}$ of DF medium containing 10\% FBS (DF-10) was overlaid on each well and $6 \mathrm{ml}$ on the $35-\mathrm{mm}$ dish and incubated in a $\mathrm{CO}_{2}$ incubator at $37^{\circ} \mathrm{C}$ overnight.

At the 0-time control, the drops in the $35-\mathrm{mm}$ dish were stained with neutral red and fixed with $10 \%$ formalin and dried. In each well of the 6-well plates, the anticancer drugs were added at final concentrations (Table I) and incubated for $24 \mathrm{~h}$. Following the removal of the medium containing the anticancer drugs, each well was rinsed twice, overlaid with serum-free culture medium (PCM-2; Nitta Gelatin Inc.) and incubated further for 7 days. Only 5-fluorouracil (5-FU) was left in the culture media for 7 days. Control drops were also cultured for 7 days without the drugs under the same condition.

After the 7-day culture period, neutral red was added to each well at a final concentration of $50 \mu \mathrm{g} / \mathrm{ml}$, and viable

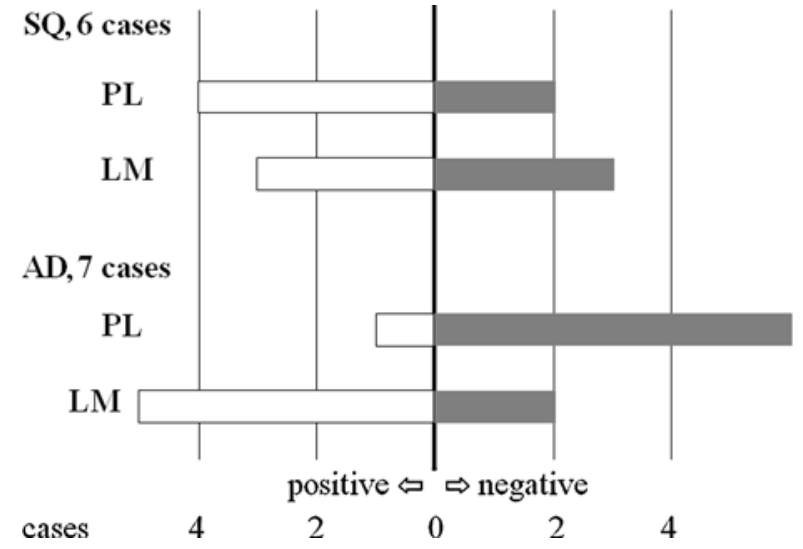

Figure 1. Chemosensitivity against 5-fluorouracil. In adenocarcinoma (AD), the number of lymph node metastases (LMs) that were assessed as positive was much higher than the number of primary lesions (PLs). SQ, squamous cell carcinoma.
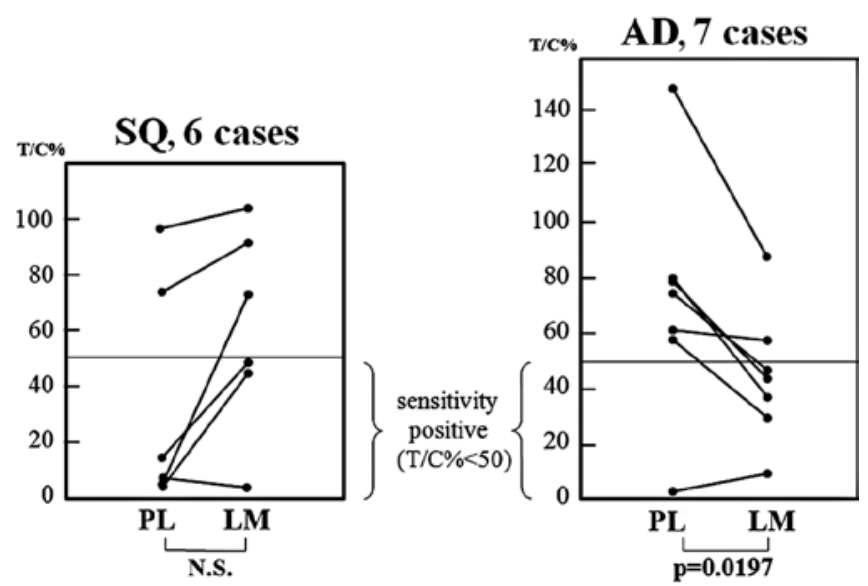

Figure 2. Proliferation rate against 5-fluorouracil (5-FU). In the adenocarcinoma (AD) cases, the T/C percentage of the lymph node metastases (LMs) was significantly lower than that of the primary lesions (PLs). Consequently, the LMs were more sensitive to 5-FU than the PLs. SQ, squamous cell carcinoma.

colonies in the droplets were stained for 1-2 h. Each droplet was fixed with $10 \%$ formalin, washed in water and dried. A video microscope (Pico Scopeman; Moritex, Tokyo, Japan), a grayscale image digitizer (LG-3; Scion Corp., MD, USA), a personal computer (Apple Power Macintosh G3; Tokyo, Japan) and modification of the NIH Image Macro-program (Primage; Toshiba Tech Corp., Tokyo, Japan) were used to measure and quantify the amount of neutral red dye taken up by the viable cells in the droplets.

When the ratio of control (7-day culture without drug) to the 0 -time control was $>0.8$, the case was regarded as assessable. When the growth rate, which was determined by the T/C ratio $(\mathrm{T}$, the signal for the viable cells in the treated group and $\mathrm{C}$, the signal in the control on day 7 ) was $<50 \%$, we regarded the case as sensitive to the drug.

Statistical analysis. Statistical significance was determined using the paired Student's t-test. Test analyses were performed 
Table II. Chemosensitivity of the squamous cell carcinoma and adenocarcinoma cases.

A, Six cases of squamous cell carcinoma

\begin{tabular}{|c|c|c|c|c|c|c|c|c|c|}
\hline Age & Gender & Stage & Origin of cells & $5-\mathrm{FU}$ & CDDP & GEM & $\mathrm{TXT}$ & VNR & SN38 \\
\hline \multirow[t]{2}{*}{75} & M & IIIA & PL & + & - & + & + & + & + \\
\hline & & & LM & - & - & - & - & + & - \\
\hline \multirow[t]{2}{*}{73} & M & IIIA & PL & + & + & + & + & + & + \\
\hline & & & LM & + & - & - & + & - & + \\
\hline \multirow[t]{2}{*}{62} & M & IIIB & PL & - & - & - & - & - & - \\
\hline & & & LM & - & + & - & - & + & - \\
\hline \multirow[t]{2}{*}{78} & M & IIA & PL & + & - & - & - & - & + \\
\hline & & & LM & + & - & - & - & + & + \\
\hline \multirow[t]{2}{*}{66} & $\mathrm{~F}$ & IIIA & PL & + & + & + & + & + & + \\
\hline & & & LM & + & + & + & + & + & + \\
\hline \multirow[t]{2}{*}{62} & M & IIIB & PL & - & - & - & - & - & - \\
\hline & & & LM & - & - & - & - & - & - \\
\hline
\end{tabular}

B, Seven cases of adenocarcinoma

\begin{tabular}{|c|c|c|c|c|c|c|c|c|c|}
\hline Age & Gender & Stage & Origin of cells & $5-\mathrm{FU}$ & CDDP & GEM & TXT & VNR & SN38 \\
\hline \multirow[t]{2}{*}{74} & $\mathrm{~F}$ & IIIA & PL & - & - & + & - & - & + \\
\hline & & & LM & + & - & - & - & - & - \\
\hline \multirow[t]{2}{*}{72} & M & IIIB & PL & - & - & - & - & - & - \\
\hline & & & $\mathrm{LM}$ & + & - & - & + & - & + \\
\hline \multirow[t]{2}{*}{60} & M & IIIA & PL & - & + & - & - & + & + \\
\hline & & & LM & + & - & - & - & + & + \\
\hline \multirow[t]{2}{*}{57} & M & IIIA & PL & + & + & + & + & + & + \\
\hline & & & $\mathrm{LM}$ & + & - & - & + & + & + \\
\hline \multirow[t]{2}{*}{49} & $\mathrm{~F}$ & IIIA & PL & - & - & - & + & + & + \\
\hline & & & LM & + & - & - & + & + & + \\
\hline \multirow[t]{2}{*}{64} & $\mathrm{M}$ & IIIB & $\mathrm{PL}$ & - & - & - & - & - & - \\
\hline & & & LM & - & - & - & - & - & - \\
\hline \multirow[t]{2}{*}{63} & M & IIIA & PL & - & - & - & - & - & - \\
\hline & & & LM & - & - & - & - & - & - \\
\hline
\end{tabular}

Patient age, in years; Stage, pathological stage of the patients according to the TNM classification of the International Union against Cancer. PL, primary lesion; LM, metastatic lymph node. +, sensitivity positive; -, negative. 5-FU, 5-fluorouracil; CDDP, cisplatin; GEM, gemcitabine; TXT, docetaxel; VNR, vinorelbine; SN38, active metabolite of irinotecan (CPT-11).

on SPSS 14.0, statistical software (SPSS Japan Inc., Tokyo, Japan).

\section{Results}

Differences in the chemosensitivity between PLs and LMs. In 3 of the 13 cases, differences in the chemosensitivity against the drugs were observed between the PL and LM, while the chemosensitivity was exactly the same for all anticancer drugs in the remaining 4 cases ( 2 cases of SQ, 2 of AD; Table II). For docetaxel, vinorelbine and SN38 (active metabolite of irinotecan), the chemosensitivity between the PL and LM was approximately the same. Specifically, the chemosensitivity to vinorelbine in the AD cases was identical (Table IIB).

Higher sensitivity of LM in adenocarcinoma to 5-FU. In 5 of the $6 \mathrm{SQ}$ cases, chemosensitivity to 5-FU was the same (Fig. 1 and Table IIA). In contrast, chemosensitivity to 5-FU differed between 2 lesions in 4 of the 7 cases with AD in which the PLs were considered to be negative with respect to sensitivity and the LMs positive (Fig. 1 and Table IIB). When the growth rates $(\mathrm{T} / \mathrm{C} \%)$ of the 2 lesions in the $\mathrm{AD}$ patients 
were compared, it was observed that the growth rates of the LM lesions were significantly lower than those of the primary lesions in the AD cases (Student's t-test) (Fig. 2).

\section{Discussion}

In the 1990s, five new agents, including two taxanes (paclitaxel and docetaxel), gemcitabine, navelbine and irinotecan, were applied in the treatment of NSCLC and were shown to produce higher response rates and longer survival (16). A randomized study of 1,207 patients showed that four platinum-based combination regimens (gemcitabine, paclitaxel, docetaxel and vinorelbine) were similarly effective with a response rate of $17-21 \%$ and a 1 -year survival rate of $31-36 \%$ in previously untreated patients with stage IIIB or IV NSCLC (17). In the event that one of these new reagents can be selected, we seek the optimal choice for each patient. In vitro drug sensitivity tests have been employed to select effective chemotherapeutic agents for a given patient (1-3), and CD-DST has been established as a simple and objective in vitro chemosensitivity assay (3). The CD-DST technique has certain advantages. Chemosensitivity can be measured directly by the shrinking of tumor size in vitro, and this method of estimating chemosensitivity can yield results in a short time. Thus, this assay has been employed in clinical investigations (7-9,11-14).

The Japanese Joint Committee of the Lung Cancer Registry compiled clinicopathological data for the NSCLC Patient Japanese Lung Cancer Registry Study for 6,644 NSCLC patients who underwent resection. In their report, the 5 -year survival rate of the entire group was $52.6 \%$, and the 5-year survival rates by pathological stage were: $79.5 \%$ for IA $(n=2,009), 60.1 \%$ for IB $(n=1,418), 59.9 \%$ for IIA $(n=232)$, $42.2 \%$ for IIB $(n=757), 29.8 \%$ for IIIA $(n=1,250), 19.3 \%$ for IIIB $(n=719)$ and $20.0 \%$ for IV $(n=259)(18)$. These results indicated the need for adjuvant therapies in order to improve the outcomes for each stage. Many adjuvant chemotherapies have been administered after complete resection to target putative dormant metastases. The International Adjuvant Lung Cancer Trial Collaborative Group reported that cisplatin-based adjuvant chemotherapy improved the 5-year survival rate of patients with completely resected NSCLC (stages I-III) (19). Adjuvant chemotherapy with uracil-tegafur improves survival among patients with completely resected pathological stage I adenocarcinoma of the lung (20). In contrast, it was reported that postoperative chemotherapy using cisplatin with vindesine was not shown to be efficacious in cases of completely resected n2 NSCLC (21). Furthermore, adjuvant radiotherapy and chemotherapy with cisplatin and etoposide did not decrease the risk of intrathoracic recurrence or prolong survival in patients with completely resected stage II or IIIA NSCLC (22). These unsatisfactory results of postoperative adjuvant chemotherapies that target the dormant disease appear to be caused by the diversity of chemosensitivity of metastatic lesions. For this reason, the drug response assay for resected specimens from PLs appears to be inadequate for the control of dormant metastatic lesions. This may be one of the reasons that in vitro chemosensitivity testing has yet to come into wide use. Chemosensitivity of the metastatic lesions as well as that of the primary lesions should be considered in order to decide upon effective agents. To evaluate this hypothesis, we compared the chemosensitivities between PLs and metastatic lesions in individual patients.

In the present study, when LM was pathologically confirmed by examination of the frozen section during surgery, specimens were obtained from the PL and LM, and in vitro drug sensitivity testing was performed. The chemosensitivities for six drugs were successfully compared in 13 NSCLC patients. As a result, the chemosensitivity of the PLs differed from that of the metastatic lymph nodes for each anticancer drug in many of the cases.

The new generation chemotherapeutic agents, gemcitabine, docetaxel, vinorelbine and SN-38, yielded the same results between the 2 lesions in most of the AD cases. Specifically, the chemosensitivity for vinorelbine was found to be concomitant in all 7 cases. In contrast, the discrepancy was more striking in the cases of SQ. In these cases, the selection of anticancer drugs based on chemosensitivity testing for the primary lesion may be insufficient for adjuvant chemotherapy targeting dormant metastatic lesions.

In $\mathrm{AD}$ cases, the chemosensitivities of the metastatic lymph nodes to 5-FU were significantly higher than those of the primary lesions. 5-FU and its derivatives may be effective for adjuvant chemotherapy after complete resection of AD. This result appears to be consistent with a study involving the efficacy of uracil-tegafur for adjuvant chemotherapy after complete resection of lung AD (20). Further investigation regarding the relationship between chemosensitivity against 5-FU and the metastatic activity of AD cells is required.

In conclusion, both primary and metastatic tumors should be examined in order to ensure maximum clinical efficacy of in vitro drug sensitivity testing for adjuvant chemotherapy after complete resection of $n 1$ and $n 2$ NSCLC.

\section{References}

1. Freeman AE and Hoffman RM: In vivo-like growth of human tumors in vitro. Proc Natl Acad Sci USA 83: 2694-2698, 1986.

2. Cole SP: Rapid chemosensitivity testing of human lung tumor cells using the MTT assay. Cancer Chemother Pharmacol 17: 259-263, 1986.

3. Kobayashi $\mathrm{H}$, Tanisaka $\mathrm{K}$, Doi O, et al: An in vitro chemosensitivity test for solid human tumors using collagen gel droplet embedded cultures. Int J Oncol 11: 449-455, 1997.

4. Shaw GL, Gazdar AF, Phelps $\mathrm{R}$, et al: Individualized chemotherapy for patients with non-small cell lung cancer determined by prospective identification of neuroendocrine markers and in vitro drug sensitivity testing. Cancer Res 53: 5181-5187, 1993.

5. Gazdar AF, Steinberg SM, Russell EK, et al: Correlation of in vitro drug-sensitivity testing with response to chemotherapy and survival in extensive-stage small cell lung cancer: a prospective clinical trial. J Natl Cancer Inst 82: 117-124, 1990.

6. Cortazar P, Gazdar AF, Woods E, et al: Survival of patients with limited-stage small cell lung cancer treated with individualized chemotherapy selected by in vitro drug sensitivity testing. Clin Cancer Res 3: 741-747, 1997.

7. Higashiyama M, Kodama K, Yokouchi H, et al: Cisplatin-based chemotherapy for postoperative recurrence in non-small cell lung cancer patients: Relation of the in vitro chemosensitive test to clinical response. Oncol Rep 8: 279-283, 2001.

8. Takamura Y, Kobayashi H, Taguchi T, et al: Prediction of chemotherapeutic response by collagen gel droplet embedded culture-drug sensitivity test in human breast cancers. Int J Cancer 98: 450-455, 2002.

9. Kikuchi T, Daigo Y, Katagiri T, et al: Expression profiles of non-small cell lung cancers on cDNA microarrays: identification of genes for prediction of lymph-node metastasis and sensitivity to anti-cancer drugs. Oncogene 22: 2192-2205, 2003. 
10. Yamaue $\mathrm{H}$, Tanimura $\mathrm{H}$, Noguchi $\mathrm{K}$, et al: Chemosensitivity testing of fresh human gastric cancer with highly purified tumour cells using the MTT assay. Br J Cancer 66: 794-799, 1992.

11. Takamura Y, Kobayashi H, Taguchi T, Motomura K, Inaji H and Noguchi S: Prediction of chemotherapeutic response by collagen gel droplet embedded culture-drug sensitivity test in human breast cancers. Int J Cancer 98: 450-455, 2002.

12. Nakahara T, Sakaeda T, Nakamura T, et al: Chemosensitivity assessed by collagen gel droplet embedded culture drug sensitivity test, and MDR1, MRP1 and MRP2 mRNA expression in human colorectal adenocarcinomas. Pharm Res 21: 406-412, 2004.

13. Nagai N, Minamikawa K, Mukai K, Hirata E, Komatsu M and Kobayashi $\mathrm{H}$ : Predicting the chemosensitivity of ovarian and uterine cancers with the collagen gel droplet culture drugsensitivity test. Anticancer Drugs 16: 525-531, 2005.

14. Shimizu T, Murata S, Mekata E, et al: Clinical potential of an antitumor drug sensitivity test and diffusion-weighted MRI in a patient with a recurrent solid pseudopapillary tumor of the pancreas. J Gastroenterol 42: 918-922, 2007.

15. Cartazar P and Johnson BE: Review of the efficacy of individualized chemotherapy selected by in vitro drug sensitivity testing for patients with cancer. J Clin Oncol 17: 1625-1631, 1999.

16. Bunn PA and Kelly K: New chemotherapeutic agents prolong survival and improve quality of life in non-small cell lung cancer: a review of the literature and future directions. Clin Cancer Res 5: 1087-1100, 1998.
17. Schiller JH, Harrington D, Belani CP, et al: Eastern Cooperative Oncology Group. Comparison of four chemotherapy regimens for advanced non-small cell lung cancer. N Engl J Med 346: 92-98, 2002

18. Goya T, Asamura H, Yoshimura H, et al: The Japanese Joint Committee of Lung Cancer Registry. Prognosis of 6644 resected non-small cell lung cancers in Japan: a Japanese Lung Cancer Registry Study. Lung Cancer 50: 227-234, 2005.

19. The International Adjuvant Lung Cancer Trial Collaborative Group: Cisplatin-based adjuvant chemotherapy in patients with completely resected non-small cell lung cancer. N Engl J Med 350: 350-360, 2004.

20. Kato H, Ichinose Y, Ohta M, et al: A randomized trial of adjuvant chemotherapy with uracil-tegafur for adenocarcinoma of the lung. N Engl J Med 350: 1713-1721, 2004.

21. Tada H, Tsuchiya R, Ichinose Y, et al: A randomized trial comparing adjuvant chemotherapy versus surgery alone for completely resected pN2 non-small cell lung cancer. Lung Cancer 43: 167-173, 2004.

22. Keller SM, Adak S, Wagner H, et al: A randomized trial of postoperative adjuvant therapy in patients with completely resected stage II or IIIA non-small cell lung cancer. N Eng J Med 343: 1217-1222, 2000. 